Editorial

\title{
Use of Design of Experiments and Manuscripts Rejection Rate
}

\author{
${ }^{1}$ Aydin Berenjian and ${ }^{2}$ Jason Ryan \\ ${ }^{I}$ School of Engineering, Faculty of Science and Engineering, \\ The University of Waikato, Hamilton 3240, New Zealand \\ ${ }^{2}$ Callaghan Innovation, 69 Gracefield Road, Lower Hutt 5040, New Zealand
}

Traditional one-variable-at-a-time method was and still is for some researchers, the general procedure to determine the optimal conditions for a chemical, biochemical or biological mechanism. According to this technique, a large number of experiments should be performed to obtain a high precision in effect assessment and also the interaction among the variables cannot be defined. To overcome the limitations of this labor-intensive, time-consuming and expensive technique, the statistical Design of Experiments (DoE) was proposed by.

DoE is recently the preferred method for optimization owing to the use of pivotal principles of statistics, randomization and replication. It is a suitable method to analyze the alteration of all factors simultaneously according to a design matrix. Therefore, the effect of each variable and also the combined effect of two or more variables can be estimated with a minimum number of experiments. With the advent of modern computing this method has become a ubiquitous tool in most research labs. While this approach is a versatile method for optimisation, its application can sometimes be haphazard or inappropriate under some conditions.

Today, a significant fraction of the submitted manuscripts are influenced by the DoE methodology and the progress in the use of these techniques in the history of biotechnology has been nothing but astonishing. Based on the SCOPUS database, over the last 10 years, the number of manuscripts with 'Design of experiment' as a keyword has tripled across the major life and physical science journals. Whilst a vast majority of these manuscripts are published in medical journals and are a standard requirement for drug efficacy testing, within the biotechnology and biochemistry journals there has been a steady increase in the number of manuscripts using DoE methodology.

An ever-increasing number of manuscripts that use DoE as a means to demonstrate the optimisation of the production of microbial compounds are being published, with a vast majority reporting incremental changes to the existing literature. However, some of the most highly cited papers have used DoE approaches. DoE software can produce subsequent number of tables/figures and consequently improve the quantity of the paper. However, this technique is only half of the whole story. The novelty of the research, its contribution to the field of study and data collection is the other half.

Rejection of a manuscript in a peer-reviewed journal is a not a pleasant experience for the authors. Scientists plan to submit their works to quality journals forgetting the severe acceptance criteria for the journal. Manuscripts depend as much on DoE software tools without any contribution to the scientific community are likely to be rejected at the initial editorial screening. Manuscripts, therefore, should more rely on high quality research that helps in understanding of the role of science and technology in solving the key scientific and societal difficulties for the benefit of mankind and environment.

\section{Conclusion}

It is still expected that the use of DoE software will continue to help scientists to produce accurate information from the data generated and further enhance the excitement about the original. However, authors must consider what degree of novelty there is through the application of DoE methodology to an existing knowledge, if the only outcome is optimisation.

\section{References}

Fisher, R.A., 1974. The Design of Experiments. 9th Edn., Hafner, New York, ISBN-10: 0028446909, pp: 248. 